\title{
Targeting PD-L1 in non-small cell lung cancer using CAR T cells
}

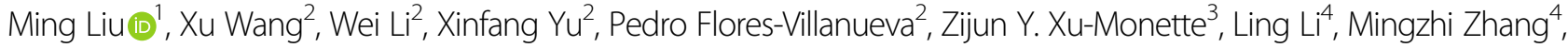 \\ Ken $\mathrm{H}$. Young $\mathbb{B}^{3}$, Xiaodong Ma $\mathbb{C}^{5}$ and Yong Li $\mathbb{B}^{2}$
}

\begin{abstract}
Antibodies against programmed cell death protein 1 (PD-1) and its ligand (PD-L1) have dramatically changed the landscape of therapies for non-small cell lung carcinoma (NSCLC); however, the majority of patients do not respond to these agents. In addition, hyperprogressive disease (HPD) develops in a larger portion of NSCLC patients treated with PD-1/PD-L1 inhibitors than in patients treated with standard chemotherapy. The use of chimeric antigen receptor (CAR) T cells has been successful to treat blood cancers but not for solid tumors like NSCLC. In this work, we constructed CAR T cells that target PD-L1 and evaluated their efficacy in NSCLC with either high or low PD-L1 expression. PD-L1-CAR T cells exhibited antigen-specific activation, cytokine production, and cytotoxic activity against PD-L1 ${ }^{\text {high }}$ NSCLC cells and xenograft tumors. Furthermore, the addition of a subtherapeutic dose of local radiotherapy improved the efficacy of PD-L1-CAR T cells against PD-L1 ${ }^{\text {low }}$ NSCLC cells and tumors. Our findings indicate that PD-L1CAR T cells represent a novel therapeutic strategy for patients with PD-L1-positive NSCLC, particularly for those who are susceptible to HPD.
\end{abstract}

\section{Introduction}

Lung cancer is the leading cause of cancer-related death in the world ${ }^{1}$. In the United States, approximately 234,000 lung cancer cases are diagnosed with 154,050 deaths annually ${ }^{1,2}$. Approximately $85 \%$ of patients with lung cancers have non-small cell lung carcinoma (NSCLC), and more than $40 \%$ of them are diagnosed with metastatic disease $^{1,3}$. Although significant progress in drug development against lung cancer has been made, the prognosis of lung cancer has not improved drastically over the past three decades ${ }^{4}$. Even with the current targeted therapies, most patients eventually experience disease relapse ${ }^{5,6}$.

Immunotherapy is a promising therapeutic approach for patients with refractory cancers. Checkpoint inhibitors

\footnotetext{
Correspondence: Ming Liu (mingliu128@hotmail.com) or

Xiaodong Ma (sciencema@hotmail.com) or Yong Li (Yong.Li@bcm.edu)

${ }^{1}$ National Clinical Research Center for Respiratory Disease, State Key Laboratory of Respiratory Disease, Guangzhou Institute of Respiratory Health, The First Affiliated Hospital of Guangzhou Medical University, 510120 Guangzhou, China ${ }^{2}$ Department of Medicine, Baylor College of Medicine, Houston, TX 77030, USA Full list of author information is available at the end of the article

These authors contributed equally: Ming Liu, Xu Wang
}

that target programmed cell death protein 1 (PD-1) or its ligand (PD-L1) have demonstrated efficacy and safety in patients with NSCLC and are becoming a standard treatment for the management of locally advanced and metastatic lung cancer. However, only approximately $20 \%$ of unselected patients with advanced NSCLC benefit from this treatment ${ }^{7}$. Furthermore, NSCLCs harboring epidermal growth factor receptor (EGFR) mutations or anaplastic lymphoma receptor tyrosine kinase $(A L K)$ rearrangements are associated with low overall response rates to PD-1/PD-L1 blockade ${ }^{8,9}$. In addition, hyperprogressive disease (HPD) represents a new pattern of progression that was recently described in cancer patients treated with PD-1/PD-L1 inhibition. A recent report shows that anti-PD-1/PD-L1 treatment accelerates tumor progression in $16 \%$ of patients with NSCLC across multiple histologies ${ }^{10,11}$. A potential mechanism is that the fragment crystallizable $(\mathrm{Fc})$ receptor of tumor-associated macrophages engages with the Fc region of the anti-PD-1 antibody to induce $\mathrm{HPD}^{12}$. Furthermore, patients with cancers that harbor mouse double minute 2 homolog 
(MDM2) amplification or EGFR mutations have increased the risk of HPD after anti-PD-1/PD-L1 treatment ${ }^{13}$. Therefore, there is an urgent need for alternative approaches to target PD-L1-positive tumors in NSCLC patients at high risk of HPD.

Chimeric antigen receptor (CAR) T-cell therapy has been successfully employed in blood tumors but not in solid tumors. The tumor microenvironment generated by myeloid-derived suppressor cells; regulatory $\mathrm{T}$ cells; immunosuppressive cytokines, such as interleukin (IL)-10 and transforming growth factor- $\beta$; and ligands for tumorexpressed T-cell inhibitory signaling receptors, such as PD-1 and CTLA-4, contribute to attenuated persistence and antitumor efficacy of CAR T cells in solid tumors ${ }^{14,15}$. The addition of checkpoint inhibitors has been applied to enhance CAR T cell efficacy ${ }^{16}$. It is shown that PD-L1 on tumor cells or on dendritic cells and macrophages in the tumor microenvironments exerts functionally significant suppressive effects on tumor immunity ${ }^{17-19}$.

High expression of PD-L1 has been found in cancer cells of NSCLC patients, and CAR T cells that secrete the anti-PD-L1 antibody have demonstrated promising efficacy in humanized mouse models ${ }^{20-22}$. In this study, we demonstrated that PD-L1-CAR T cells have substantial antitumor activity in vitro and lead to prolonged remission for PD-L1 $1^{\text {high }}$ NSCLC xenograft tumors in mice. In addition, radiotherapy exhibited synergistic activity with PD-L1-CAR $T$ cells, potentially by allowing the migration of CAR $\mathrm{T}$ cells to tumors generated from PD-L1 ${ }^{\text {low }}$ NSCLC cells. Our findings provide preclinical evidence to support PD-L1 targeting by CAR T cells to treat NSCLC and potentially other types of solid malignancies.

\section{Material and methods \\ Cell lines and culture}

Human NSCLC EGFR-wild type cell lines A549 and H1299, EGFR-mutant cell lines HCC827 (del E746-A750) and H1975 (L858R and T790M), and normal bronchial epithelial cell line (BEAS-2B) were purchased from ATCC (Manassas, VA). The NSCLC EGFR-mutant cell line PC9 (del E746-A750) was obtained as described previously ${ }^{23}$. These cell lines were maintained in RPMI-1640 (Gibco, Gaithersburg, MD) supplemented with $10 \%$ heatinactivated fetal bovine serum (Gibco) and $1 \%(\mathrm{v} / \mathrm{v})$ penicillin/streptomycin in a humidified incubator with $5 \% \mathrm{CO}_{2}$ at $37^{\circ} \mathrm{C}$. All cells were transduced with firefly luciferase (Fluc) via lentiviral transduction, and blasticidin selection was utilized to set up stable luciferase-expressing cell lines.

\section{CAR construction, lentiviral vector production, and T cell transduction}

The PD-L1-CAR, encoding single-chain variable fragment (scFv) against the human PD-L1, a CD8 hinge and transmembrane domain, 4-1BB co-stimulatory domain, and $\mathrm{CD} 3 \zeta$ signaling domain, were totally synthesized and cloned into a third-generation lentiviral plasmid backbone with a human elongation factor $1 \alpha(E F-1 \alpha)$ promoter. PD$\mathrm{L} 1 \mathrm{scFv}$ is derived from atezolizumab, a fully humanized, engineered monoclonal antibody of IgG1 isotype against PD-L1 (sold as Tecentriq ${ }^{\circledR}$ by Roche). A CD19-CAR with the same structure was used as a control. CD19 scFv is derived from mouse monoclonal antibody FMC-63 (GenBank ID: HM852952.1). PD-L1-CAR-encoding and CD19-CAR-encoding lentiviral supernatants were produced via transient transduction of the $293 \mathrm{~T}$ cell line as described $^{24} \cdot \mathrm{CD}^{+} / \mathrm{CD}^{+} / \mathrm{CD}^{+} \mathrm{T}$ cells were isolated from leukopaks of healthy volunteer donors (Gulf Coast Regional Blood Center, Houston, TX) using EasySep ${ }^{\mathrm{TM}}$ Human CD3/CD4/CD8 Positive Selection Kit (Stem Cell Technologies, Vancouver, Canada). Isolated human $\mathrm{T}$ cells were then activated by anti-CD3/CD28 beads (Life Technologies, Carlsbad, CA) in a cell-to-bead ratio of 1:3 with $200 \mathrm{IU} / \mathrm{ml}$ IL-2 in CTS $^{\mathrm{TM}}$ OpTmizer $^{\mathrm{TM}} \mathrm{T}$ Cell Expansion medium (Life Technologies). After $24 \mathrm{~h}$, activated $\mathrm{T}$ cells were transduced by the PD-L1-CAR or CD19-CAR. Medium with IL-2 was refreshed every 2-3 days. Each ensuing cellular or animal experiment was performed using $\mathrm{T}$ cells from at least two different donors. In all figure legends, the data were obtained using $\mathrm{T}$ cells from one donor only with technical triplicates.

\section{Cellular cytotoxicity assay}

The cytotoxicity of $\mathrm{T}$ cells was assessed using a luciferasebased assay as previously described ${ }^{25}$. Stable Flucexpressing tumor cells $(20,000$ cells per well) were coincubated with PD-L1- or CD19-CAR T cells for 4 or $20 \mathrm{~h}$ at effector-to-target (E:T) ratios from 10:1 to 1:4. The onestep glow assay kit (Thermo Fisher Scientific, Waltham, MA) was used to measure residual luciferase activity from the remaining tumor target cells, and lysis was calculated as follows: $\%$ lysis $=100-$ (Fluc from CAR-T-treated wells) / (Fluc from untreated target cells) $\times 100$.

\section{Cytokine secretion assay}

Cytokine production by CAR T cells in vitro was evaluated following the co-incubation of CAR $\mathrm{T}$ cells with tumor cells at a 2:1 ratio for $20 \mathrm{~h}$. Supernatants were harvested, and cytokine levels were measured using Human DuoSet ELISA kits (IL-2, tumor necrosis factor $[\mathrm{TNF}]-\alpha$, and interferon $[\mathrm{IFN}]-\gamma, \quad \mathrm{R} \& \mathrm{D}$ Systems, Minneapolis, MN).

\section{Flow cytometry}

Expression levels of PD-L1 and other cell surface markers on tumor cells and $\mathrm{T}$ cells were measured using flow cytometry. CAR T cells were collected from cultures and detected with monoclonal antibodies against human $\mathrm{CD} 3$, CD4, CD8, TIM3, CD45RA, CD62L, PD-L1, and PD-1 
(Biolegend, San Diego, CA) according to the manufacturers' instructions. PD-L1-CAR expression was detected using an indirect method with biotinylated protein L and a streptavidin-coupled PE antibody (Becton Dickinson, Franklin Lakes, NJ) ${ }^{26}$. Fluorescence was assessed using BD Accuri ${ }^{\mathrm{TM}}$ C6 Plus or LSRFortessa instruments (BD Biosciences, San Jose, CA), and the data were analyzed using FlowJo v10 (Tree Star, Ashland, OR).

\section{Xenograft mouse model}

All animal procedures were performed in accordance with our Institutional Animal Care and Use Committee requirements under an approved protocol. Female NOD. Cg-Prkdc ${ }^{\text {scid }}$ Il2rg ${ }^{\text {tm1Wjil }} /$ SzJ (NSG, The Jackson Laboratory, Bar Harbor, ME) mice aged 6-8 weeks were maintained in a pathogen-free barrier facility. Mice were inoculated subcutaneously with $1.0 \times 10^{6}$ H1975-Fluc cells, $3 \times 10^{6}$ HCC827-Fluc cells, $5.0 \times 10^{6}$ A549-Fluc cells, or $2.0 \times 10^{6}$ H1299-Fluc cells. Animals were treated with $5 \times 10^{6}$ CAR $\mathrm{T}$ cells twice via tail vein injection on day 7 and 10 posttumor cell inoculation. Tumor progression was monitored using an IVIS Spectrum in vivo Imaging System (Perkin Elmer, Waltham, MA). Tumor volumes were calculated according to the formula: $V=1 / 2$ (length $\times$ width $^{2}$ ).

\section{Histology immunohistochemistry analyses}

Tissue samples were collected and stained followed the manufacturer's protocol. In brief, deparaffinized and rehydrated sections were treated for antigen retrieval using sodium citrate buffer, blocked with normal goat serum for $30 \mathrm{~min}$ at room temperature, and then incubated with primary antibody against CD3 (ab16669, Abcam, Cambridge, UK), PD-L1(ab228462, Abcam), Ki67 (D2H10, Cell Signaling Technology, Danvers, MA) at $4{ }^{\circ} \mathrm{C}$ overnight. Slides were incubated with secondary antibodies, counterstained with hematoxylin, and visualized by the Ultra Vision Detection System (Thermo Fisher Scientific). Signal intensity was scored by two independent observers who were blind to the experimental groups.

\section{Statistical analysis}

The data were presented as means \pm standard error (SEM). Two independent groups were analyzed using Student's $t$-test, while the statistical comparison between multiple groups was performed using two-way repeatedmeasures ANOVA. $p$-values $\leq 0.05$ were considered statistically significant. All statistical analyses were performed using GraphPad Prism v7.0 (GraphPad Software, San Diego, CA).

\section{Results}

\section{Generation of PD-L1-CAR-expressing T cells}

To generate anti-PD-L1-CAR T cells, we constructed a CAR expression plasmid that encodes an anti-PD-L1 scFv, a CD8 hinge, a transmembrane domain in tandem with 4$1 \mathrm{BB}$ intracellular signaling domain, and a $\mathrm{CD} 3 \zeta$ motif (Fig. 1a). A CD19-CAR with an anti-CD19 scFv was used as a control. The expression level of the CAR was evaluated by flow cytometry 5 days of post-transduction. An average of $55.6 \%$ of the PD-L1-CAR T cells and $59.3 \%$ of the CD19-CAR $\mathrm{T}$ cells were scFv-positive, indicating a high transduction efficiency (Fig. 1b, Supplementary Fig. 1a). There was no significant difference in cell viability between PD-L1-CAR T and CD19-CAR T cells on day 7 and 14 post-transduction (Fig. 1c). CD19-CAR and PDL1-CAR $\mathrm{T}$ cells displayed a similar expansion tendency after priming with anti-CD3/CD28 beads for $14 \mathrm{~d}(>50$ fold expansion; Fig. 1d). Importantly, both $\mathrm{CD}^{+}$and $\mathrm{CD}^{+}$PD-L1-CAR $\mathrm{T}$ cells, upon anti-CD3/CD28 bead stimulation, were expanded to more than $5 \times 10^{7}$ cells from an initial $1 \times 10^{6}$ transduced primary $\mathrm{T}$ cells (Supplementary Fig. 2a). To investigate the expression level of different cell markers at an early and late stage of culture, we examined $\mathrm{T}$-cell markers for lineage, immunosuppression, and memory phenotypes. On day 7, both PD-L1CAR and CD19-CAR T cells were positive for CD3 (96.8 and $97.8 \%$, respectively), CD4 (68.3 and $66.8 \%$, respectively), and CD8 (26.4 and 29.1\%, respectively). For PDL1-CAR T cells, $28.5 \%, 3.9 \%$, and $1.4 \%$ of them were positive for PD-1, PD-L1, and TIM3, respectively, on day 7. During PD-L1 CAR T cell expansion, the expression of PD-1 on these cells was higher at day 14 than that at day 7 (Fig. 1e, Supplementary Fig. 1b); however, there was no differences in fractions of central memory $\mathrm{T}$ cells $\left(\mathrm{CD} 45 \mathrm{RA}^{-} \mathrm{CD} 6 \mathrm{~L}^{+}, T_{\mathrm{cm}}\right)$, stem cell-like memory $\mathrm{T}$ cells (CD45RA $\left.{ }^{+} \mathrm{CD}_{62 \mathrm{~L}^{+}}, T_{\mathrm{scm}}\right)$, and effector memory $\mathrm{T}$ cells $\left(\mathrm{CD}_{4}\right.$ RA ${ }^{+} \mathrm{CD} 2 \mathrm{~L}^{-}, T_{\mathrm{em}}$ ) between day 14 and day 7 (Fig. 1f, Supplementary Fig. 1c). In summary, after 14 days of culture, $\mathrm{CD}^{+}, \mathrm{CD}^{+}$, and $\mathrm{CD} 8^{+}$PD-L1-CAR T cells expanded well and contained both effector and central memory cell populations.

\section{PD-L1-CAR T cells exhibit robust effector functions against PD-L1 ${ }^{\text {high }}$ NSCLC cells in vitro}

PD-L1 was highly expressed on H1975, HCC827, and PC9 cells, which carry mutant EGFR, whereas A549, H1299 (both with wild-type EGFR), and an immortalized bronchial cell line (BEAS-2B) showed lower PD-L1 expression (Fig. 2a). To assess the antitumor efficacy of PD-L1-CAR T cells, we performed cytotoxicity assays against these NSCLC cell lines and BEAS-2B. PD-L1-CAR $\mathrm{T}$ cells and CD19-CAR $\mathrm{T}$ cells were co-cultured at selected effector-to-target ratios for 4 or $20 \mathrm{~h}$. Compared to CD19-CAR T cells, PD-L1-CAR T cells showed significantly stronger cytotoxic activity for $\mathrm{PD}-\mathrm{L} 1^{\text {high }}$ cell lines (H1975, HCC827, and PC9) but not for PD-L1 ${ }^{\text {low }}$ cell lines (A549, H1299, and BEAS-2B; Fig. 2b). Furthermore, both $\mathrm{CD}^{+}$and $\mathrm{CD}^{+}$PD-L1-CAR $\mathrm{T}$ cells 


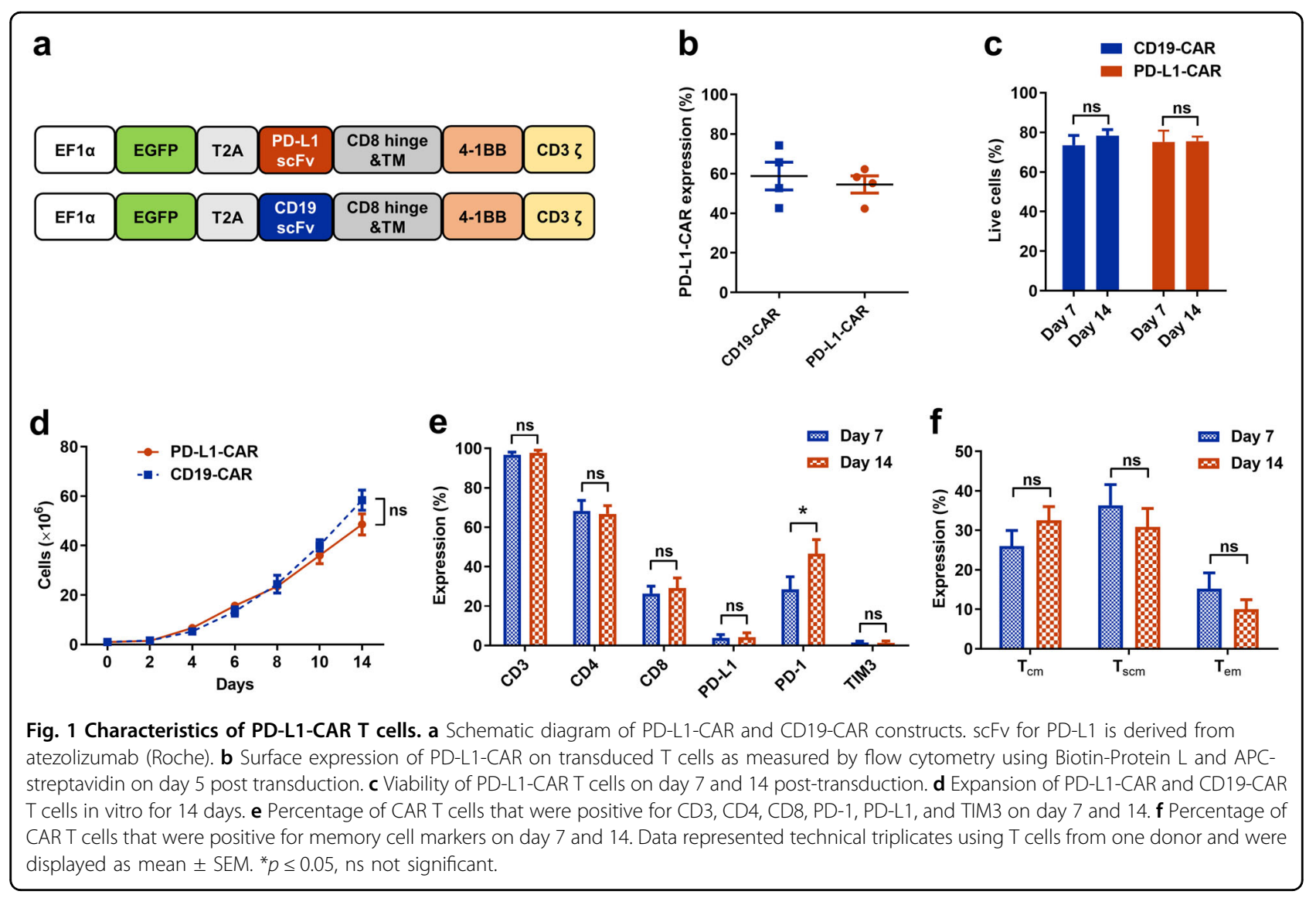

exhibited efficient cytotoxicity against PD-L1 ${ }^{\text {high }}$ tumor cells (Supplementary Fig. 2b). PD-L1-CAR T cells also demonstrated antigen-specific production of cytokines IL2 , IFN- $\gamma$, and TNF- $\alpha$ when incubated with PD-L1 ${ }^{\text {high }}$ tumor cell lines HCC827 and H1975 in vitro (Fig. 2c).

\section{PD-L1-CAR T cells eradicate PD-L1 ${ }^{\text {high }}$ NSCLC tumors in vivo}

To determine the efficacy of PD-L1 CAR T cells in vivo, we inoculated NSG mice subcutaneously with H1975Fluc followed by two doses of PD-L1-CAR or CD19-CAR $\mathrm{T}$ cells via tail vein on day 7 and 10. Tumor xenografts were monitored via bioluminescence imaging weekly (Fig. 3a). Serial imaging of luminescence showed that PD-L1CAR T cells dramatically decreased tumor burden compared with CD19-CAR T cells (Fig. 3b). The radiance of the tumors was significant reduced after PD-L1-CAR Tcell treatment (Fig. 3c). Flow cytometry analysis on cells extracted from tumors day 28 post-inoculation indicated that the expression of PD-L1 was significantly decreased in tumor cells upon PD-L1-CAR T cell treatment (Fig. 3d). This result was corroborated by immunohistochemical (IHC) analysis of PD-L1 in tumor sections (Fig. 3e). Fewer Ki67-positive cells were observed in tumors treated with PD-L1-CAR T cells than that with the control CD19CAR $T$ treatment (Fig. 3e). No significant differences in tissue morphology were found in the spleens and lungs from mice treated with PD-L1-CAR or CD19-CAR T cells (Fig. 3f).

We next tested PD-L1-CAR T cells in treating another PD-L1 ${ }^{\text {high }}$, EGFR-mutant tumor cell line HCC827. Tumor xenografts (at the right flank of mice) were monitored via bioluminescence imaging weekly after injection of CAR $\mathrm{T}$ cells (Fig. 4a). On day 28, HCC827-Fluc tumor cells were almost completely eliminated by PD-L1-CAR T cell treatment, and no obvious tumor was observed during the next 6 weeks (Fig. 4b, c). The control CD19-CAR T cells exhibited no tumorcidic activity. We re-challenged the mice treated with PD-L1-CAR T cells by injecting another dose of HCC827-Fluc cells on day 70 on the contralateral flank of the animals. Comparable and sustained antitumor activity was observed in the re-challenged group (Fig. 4b, d), while tumor growth was observed in a new control animal cohort treated with CD19-CAR $\mathrm{T}$ cells. This suggests that PD-L1-CAR T cells had sustained antitumor activity. Collectively, these results demonstrated that PDL1-CAR T cells exhibited a significant antitumor effect against PD-L1 ${ }^{\text {high }}$ NSCLC cells in vivo. 


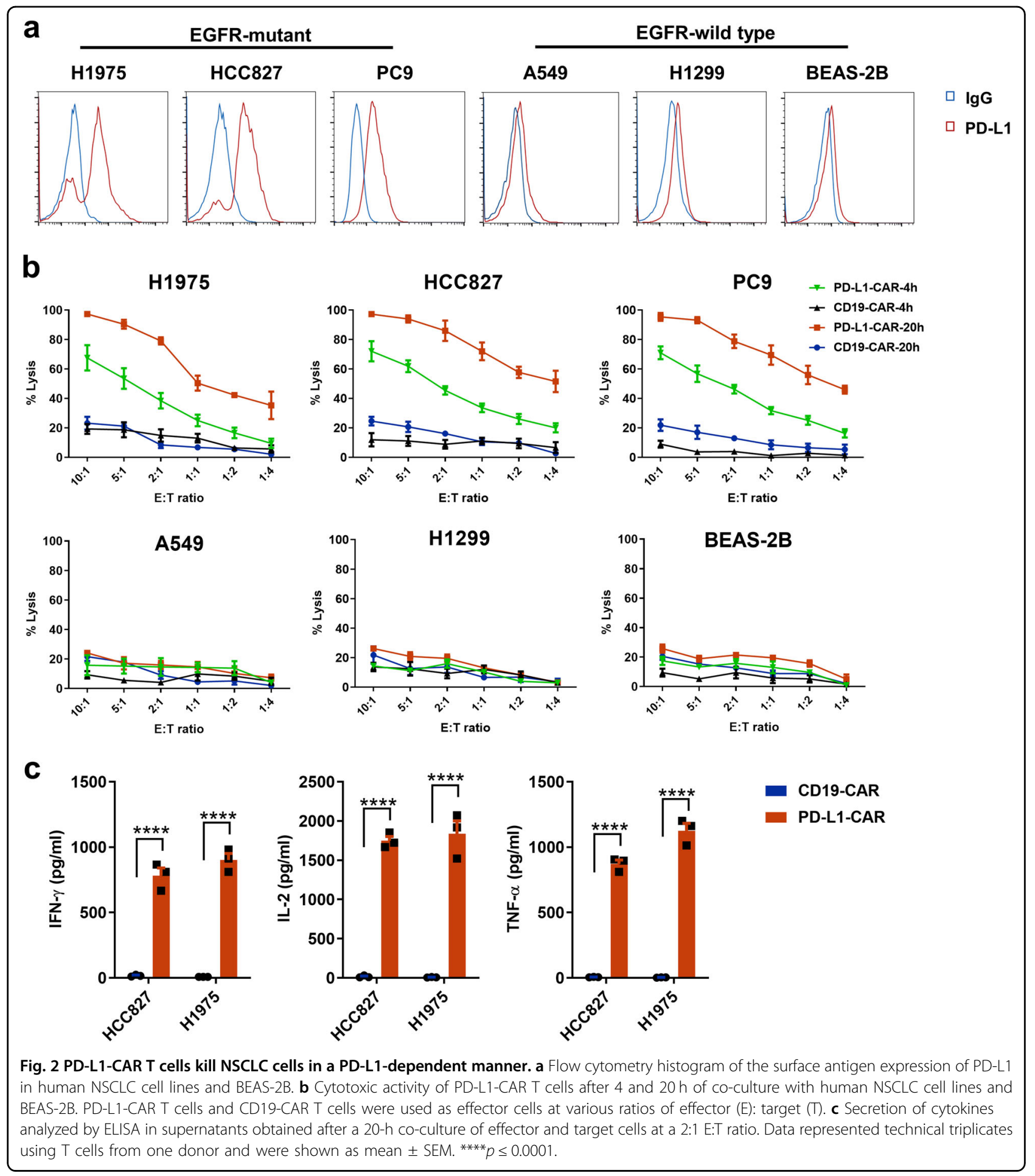

Evaluation of the role of IFN $-\gamma$ and irradiation in promoting the antitumor activity of PD-L1-CAR T cells against PDL1 ${ }^{\text {low }}$ NSCLC cells

In order to improve the efficacy of PD-L1-CAR T-cell treatment in PD-L1 ${ }^{\text {low }}$ NSCLC cells, we sought to induce PD-L1 expression in NSCLC cells. We treated cells with $5 \mathrm{ng} / \mathrm{ml}$ IFN- $\gamma$ and analyzed PD-L1 expression. A significant increase in the expression of PD-L1 was observed in all NSCLC cell lines except BEAS-2B (Supplementary Fig. 3a). Next, A549 and HCC827 cells were pre-treated with $5 \mathrm{ng} / \mathrm{ml}$ IFN- $\gamma$ for $24 \mathrm{~h}$ followed by PD-L1-CAR $\mathrm{T}$ cells or CD19-CAR $\mathrm{T}$ cells for an 


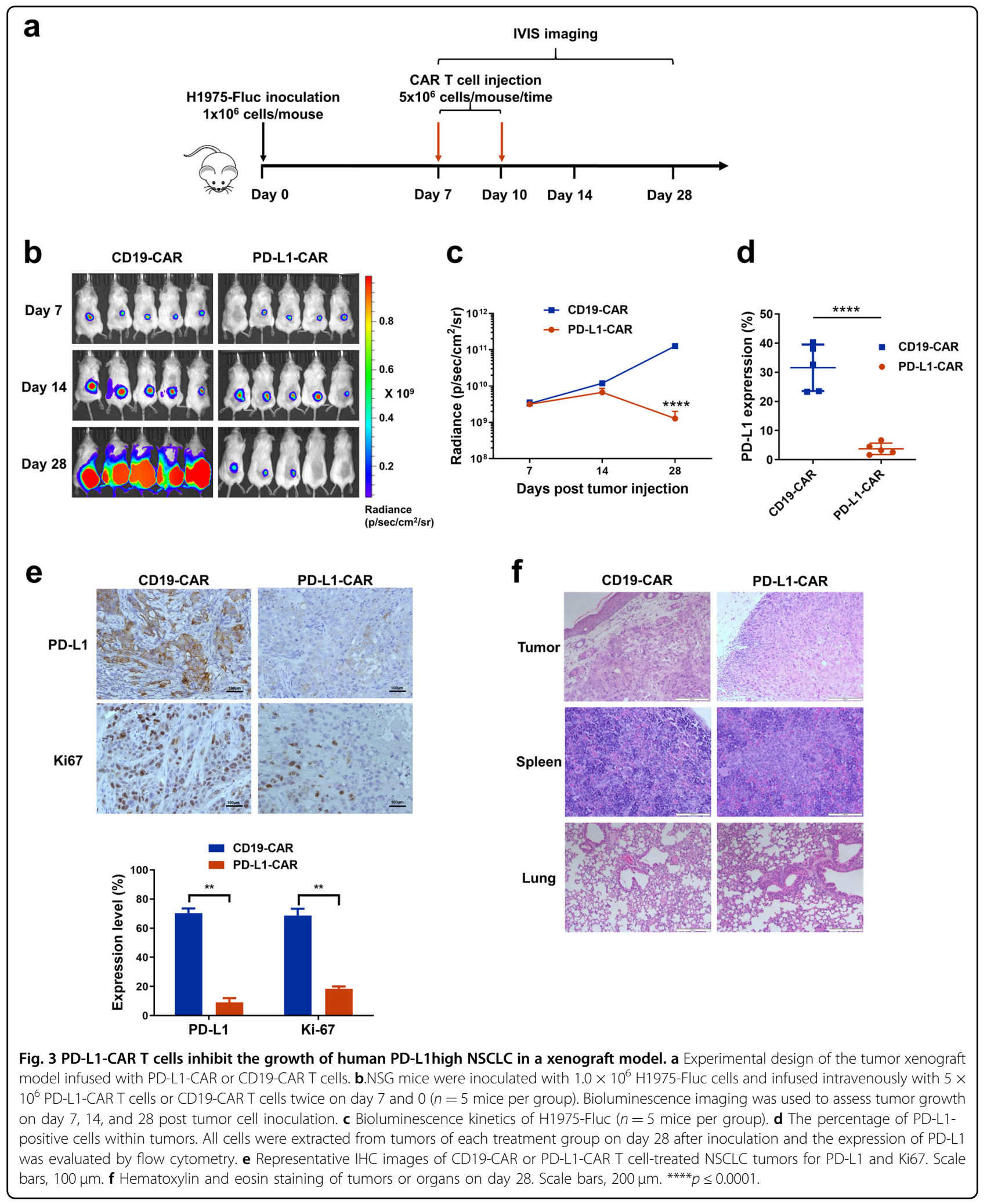

additional 4 or $20 \mathrm{~h}$. We found no significant difference in tumor cell lysis with or without IFN- $\gamma$ pre-treatment (Supplementary Fig. 3b).
Radiotherapy, a lung cancer treatment option that directly induces tumor cell apoptotic death and enhances tumor-specific immunity, has been shown to upregulate 




PD-L1 expression in tumor cells and improve the efficacy of anti-PD-1/PD-L1 therapy ${ }^{27,28}$. Incomplete tumor eradication by radiation-induced adaptive immunity is partially due to the engagement of negative regulatory pathways, such as the PD-L1/PD-1 axis ${ }^{29}$. We applied 5 Gy irradiation to BEAS-2B, A549, H1299, H1975, HCC827, and PC9 cell lines and found moderately and statistically significant increased expression of PD-L1 in PD-L1 ${ }^{\text {low }}$ A549 cells, but not in H1299 cells (Fig. 5a, b). However, radiation resulted in significant upregulation of PD-L1 in BEAS-2B cells. Irradiation with 5 Gy prior to the addition of PD-L1-CAR $\mathrm{T}$ cells significantly increased cytolysis of A549 cells, whereas no significant difference in cytolysis was found with CD19-CAR T cells (Fig. 5c). In mice xenografted with PD-L1 ${ }^{\text {low }}$ A549 and H1299 cells and then treated with CAR T cells and 5 Gy localized irradiation (Fig. 6a; Fig. S4a), irradiation alone had no effect on tumorigenesis but increased the antitumor activity of PD-L1-CAR T cells (Figs. 6b, c; S4b). Notably, increased PD-L1 expression was observed by IHC in A549 tumors $72 \mathrm{~h}$ post-irradiation (Fig. 6d). Irradiation also increased tumor-infiltrating CAR T cells and reduced cell proliferation for both A549 and H1299 tumors (Figs. 6e; S4c). The combination of radiation and PD-L1-CAR-T cells resulted in fewer proliferative tumor cells than either agent alone (Figs. 6f; S4c). The reduction of tumorigenesis for both A549 and H1299 treated by the combination is relatively moderate compared to that for $\mathrm{H} 1975$ and HCC 827 by CAR T cells alone (Figs. 3, 4). Nonetheless, these data support the notion that the combination of localized irradiation and PD-L1-CAR $\mathrm{T}$ cells attenuates the growth of tumors from PD-L1 ${ }^{\text {low }}$ NSCLC cells.

\section{Discussion}

$E G F R$ mutation is a frequent cancer-driving event in NSCLC, occurring in about $40-50 \%$ of cases in Asia and $20-30 \%$ in the United $\operatorname{States}^{30}$. In patients with advanced, EGFR-mutant NSCLC, PD-L1 expression is found in more than $50 \%$ of cases ${ }^{31}$, but EGFR-mutant NSCLC has a poor response to anti-PD-1/PD-L1 treatment ${ }^{32}$. In addition, a significant portion of NSCLC patients develop HPD after anti-PD-1/PD-L1 therapy ${ }^{10,11}$, and the EGFR mutation is a proposed risk factor for HPD ${ }^{13}$. In this work, we explored PD-L1-CAR T-cell therapy as an alternative treatment approach for NSCLC with PD-L1 ${ }^{\text {high }}$ and EGFR mutant phenotypes (for example, PD-L1 expression assessed to be $\geq 50 \%$ tumor proportion score). We showed that EGFR-mutant NSCLC cells such as HCC827, H1975, and PC9 expressed high levels of PD-L1 and PD-L1-CAR $\mathrm{T}$ cells have strong cytotoxic activity against these cells and xenograft tumors.

PD-L1 is induced in tumors and in cultured tumor cells by IFN- $\gamma$ exposure. However, in present work, IFN- $\gamma$ failed to increase PD-L1-CAR T cells efficacy against PDL1 ${ }^{\text {low }}$ NSCLC cells. This could be a result of deficiency of IFN- $\gamma$ treatment dose and duration. Given the transient nature of PD-L1 induction by IFN- $\gamma$, future optimization by biologics or compounds should be considered for long- 


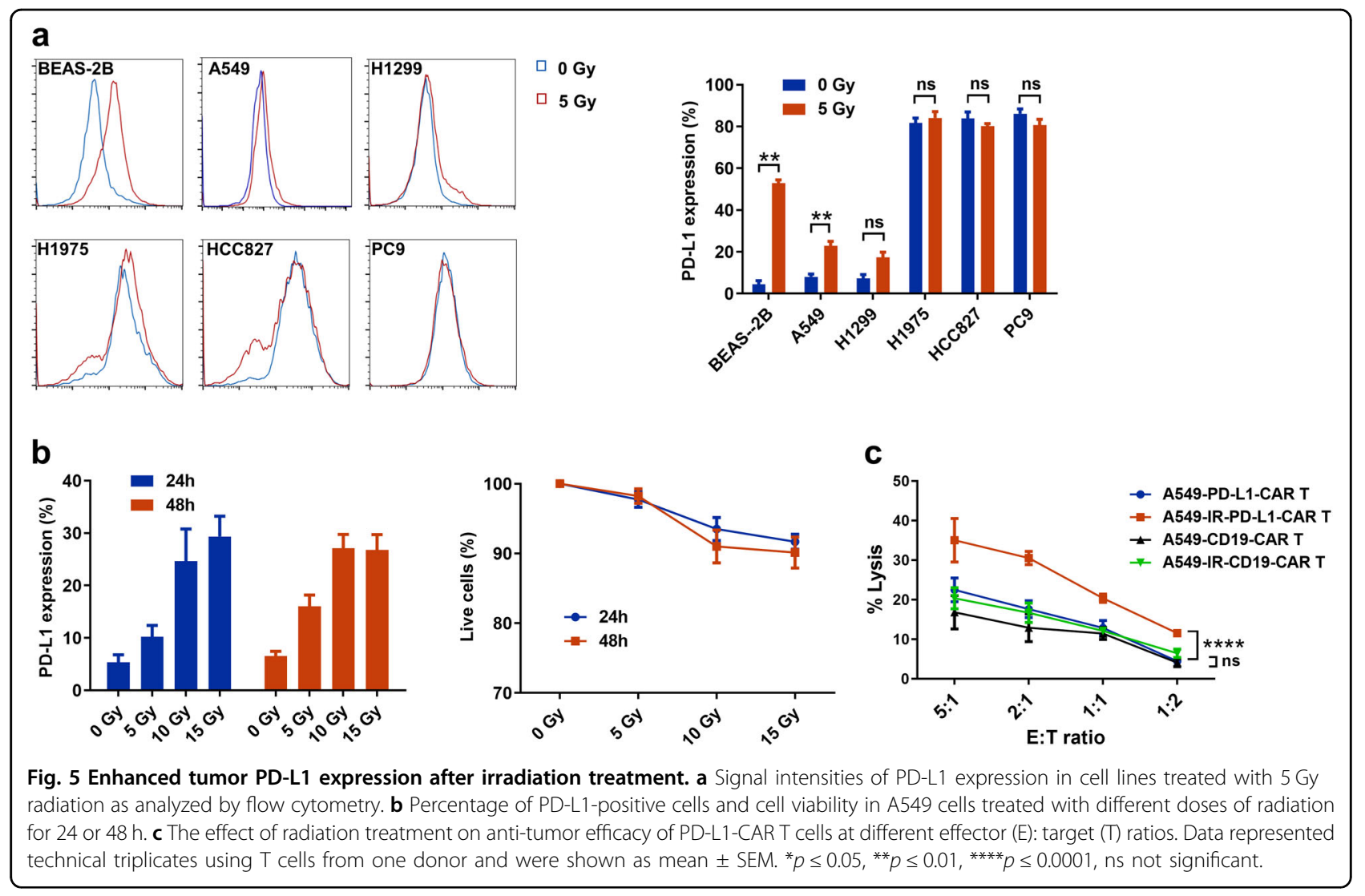

term stimulation of PD-L1 expression without attenuating $\mathrm{T}$ cells function. Substantial evidence has shown that the combination of radiotherapy and immunotherapy is more effective than monotherapy ${ }^{29}$. Preclinical studies have demonstrated that PD-L1 expression is upregulated on tumor cells after radiotherapy, resulting in a synergistically enhanced antitumor effect of irradiation and PD-L1 blockade $^{29}$. Patients receiving radiotherapy before antiPD-1 treatment have a better prognosis than those that receive anti-PD-1 alone. Another study indicated that this synergy stems from type I interferon production induced by radiotherapy ${ }^{33}$. Our results show that radiation improves the killing ability of PD-L1-CAR T cells against NSCLC xenograft tumors that otherwise express low levels of PD-L1. This is likely due to the increased CAR T cell infiltration into the tumors, rather than radiationmediated elevation of PD-L1 expression on tumor cells. These data could broaden the potential clinical applications of PD-L1-CAR T cells for the treatment of NSCLC and other solid tumors.

Among main difficulties of targeting solid tumors using CAR $\mathrm{T}$ cells is the lack of tumor-specific membrane antigens or antigens that are shared by dispensable cell types such as B cells, which prompt many to use suboptimal targets such as PD-L1. Beyond placenta, tonsil, and macrophages in lung and liver, PD-L1 protein is not expressed in steady-state normal human tissues, although the mRNA of $P D-L 1$ is present in many tissues or cells ${ }^{34-}$ ${ }^{37}$. In mice, CAR T cells targeting Pd-11 were effective in slowing tumorigenesis in a B16 syngeneic mouse model ${ }^{38}$; the toxicity of CAR $\mathrm{T}$ cells towards Pd-11-expressing mouse tissues was not directly addressed, although Cd11b-positive cells were the most adversely effected lymphocytes by anti-Pd-11 CAR $\mathrm{T}$ cells ${ }^{38}$. In the present study, we only used the NSG model to test the antihuman PD-L1-CAR $\mathrm{T}$ cell therapies against human tumors without evaluating the on-target and off-target toxicity in vivo. Radiation may augment the on-target toxicity of PD-L1 CAR T cells in humans as it clearly increases the expression of PD-L1 in BEAS-2B cells. We plan to assess the safety of PD-L1-CAR-T cells by using immunocompetent mouse models before considering phase 1 clinical trials.

In conclusion, PD-L1-CAR $\mathrm{T}$ cells are a promising therapeutic strategy for NSCLC with PD-L $1^{\text {high }}$ and EGFR mutation. Furthermore, the addition of radiation sensitizes PD-L1 ${ }^{\text {low }}$ EGFR-wild type NSCLC to PD-L1-CAR $\mathrm{T}$ cells. PD-L1-CAR T cells thus represent a novel therapeutic option for NSCLC patients who are susceptible to HPD. 


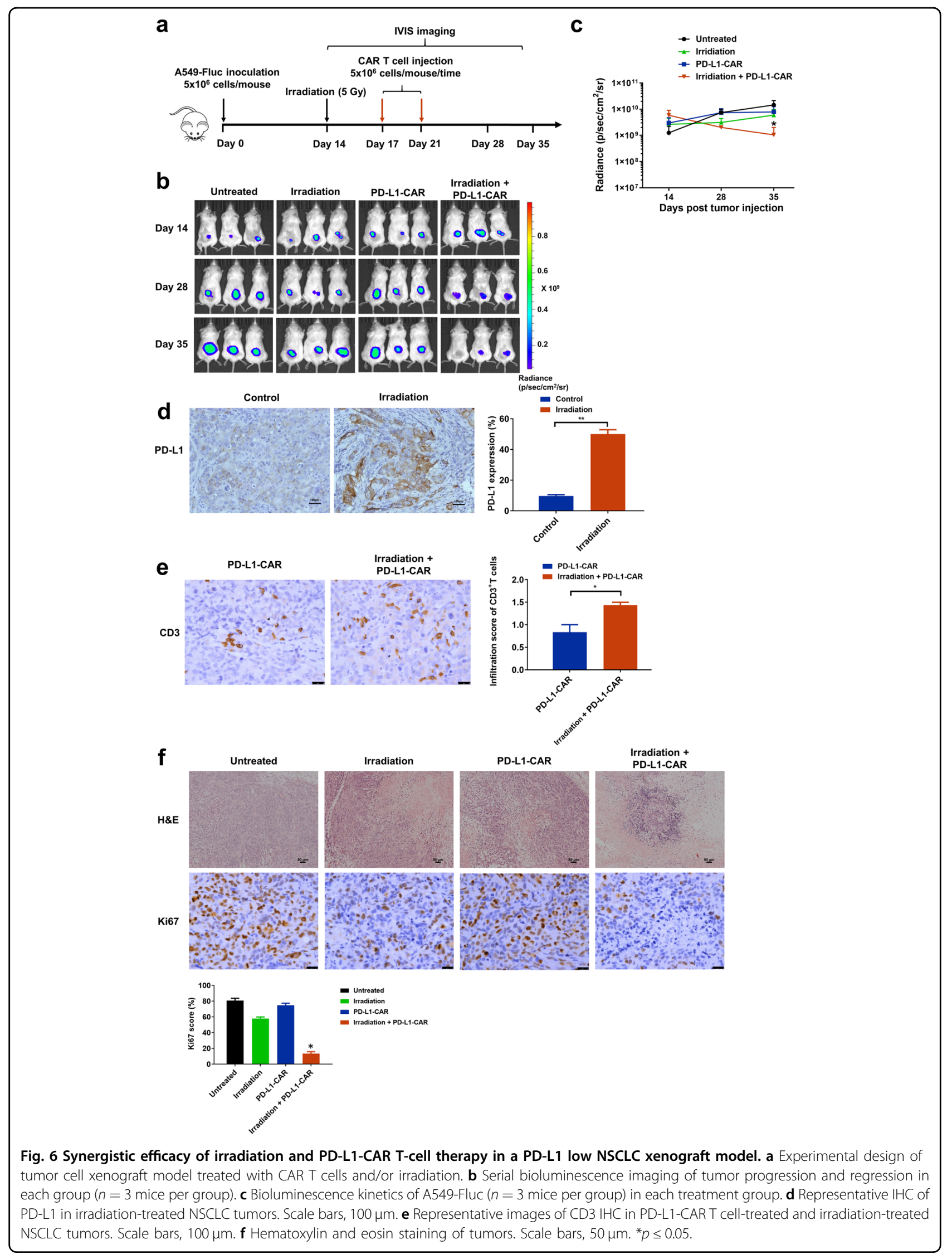




\section{Acknowledgements}

M.L., X.W., and Y.L. designed the research. All authors performed experiments and/or contributed to data analyses. M.L., X.W., and Y.L. wrote the manuscript, and all authors provided critical review and revisions and approved the final version of the manuscript. All data and materials supporting the conclusion of this study have been included within the article and the supplemental data. Animal experiments are approved by our Institutional Animal Care and Use Committees. There is no human subject participation. This study is supported by National Natural Science Foundation of China (81773249 to X.M.), Key Realm R\&D Program of Guangdong Province (2019B030335001 to X.M.). Y.L. is supported in part by the Cancer Prevention and Research Institute of Texas (RR190043) and by the Superfund Hazardous Substance Research and Training Program from National Institutes of Health (P42 ES027725).

\section{Author details}

'National Clinical Research Center for Respiratory Disease, State Key Laboratory of Respiratory Disease, Guangzhou Institute of Respiratory Health, The First Affiliated Hospital of Guangzhou Medical University, 510120 Guangzhou, China. ${ }^{2}$ Department of Medicine, Baylor College of Medicine, Houston, TX 77030, USA. ${ }^{3}$ Department of Pathology, Division of Hematopathology, Duke University Medical Center, Durham, NC, USA27708. ${ }^{4}$ Department of Oncology, The First Affiliated Hospital of Zhengzhou University, Lymphoma Diagnosis and Treatment Center of Henan Province, 450052 Zhengzhou, China. ${ }^{5}$ Institute for Brain Research and Rehabilitation, South China Normal University, 510631 Guangzhou, China

\section{Conflict of interest}

The authors declare that they have no conflict of interest.

\section{Publisher's note}

Springer Nature remains neutral with regard to jurisdictional claims in published maps and institutional affiliations.

Supplementary Information accompanies this paper at (https://doi.org/ 10.1038/s41389-020-00257-z).

Received: 28 January 2020 Revised: 8 July 2020 Accepted: 29 July 2020 Published online: 13 August 2020

\section{References}

1. Bray, F. et al. Global cancer statistics 2018: GLOBOCAN estimates of incidence and mortality worldwide for 36 cancers in 185 countries. Cancer J. Clin. https:// doi.org/10.3322/caac.21492 (2018).

2. Boloker, G., Wang, C. \& Zhang, J. Updated statistics of lung and bronchus cancer in United States (2018). J. Thorac. Dis. 10, 1158-1161 (2018).

3. Tamura, T. et al. Specific organ metastases and survival in metastatic nonsmall-cell lung cancer. Mol. Clin. Oncol. 3, 217-221 (2015).

4. Lanuti, M. et al. Observations in lung cancer over multiple decades: an analysis of outcomes and cost at a single high-volume institution. Eur. J. Cardio Thorac. Surg. 46, 254-261 (2014).

5. Bui, K. T., Cooper, W. A., Kao, S. \& Boyer, M. Targeted molecular treatments in non-small cell lung cancer: a clinical guide for oncologists. J. Clin. Med. https:// doi.org/10.3390/jcm7080192 (2018).

6. Pakkala, S. \& Ramalingam, S. S. Personalized therapy for lung cancer: striking a moving target. JCl Insight https://doi.org/10.1172/jci. insight.120858 (2018).

7. Socinski, M. A. et al. Atezolizumab for first-line treatment of metastatic nonsquamous NSCLC. N. Engl. J. Med. 378, 2288-2301 (2018).

8. Gainor, J. F. et al. EGFR mutations and ALK rearrangements are associated with low response rates to PD-1 pathway blockade in non-small cell lung cancer: a retrospective analysis. Clin. Cancer Res. 22, 4585-4593 (2016).

9. Dong, Z. Y. et al. EGFR mutation correlates with uninflamed phenotype and weak immunogenicity, causing impaired response to PD-1 blockade in nonsmall cell lung cancer. Oncoimmunology 6, e1356145 (2017).

10. Ferrara, R. et al. Hyperprogressive disease in patients with advanced non-small cell lung cancer treated with PD-1/PD-L1 inhibitors or with single-agent chemotherapy. JAMA Oncol. https://doi.org/10.1001/jamaoncol.2018.3676 (2018).

11. Champiat, S. et al. Hyperprogressive disease: recognizing a novel pattern to improve patient management. Nat. Rev. Clin. Oncol. https://doi.org/10.1038/ s41571-018-0111-2 (2018).

12. Lo Russo, G. et al. Antibody-Fc/FcR interaction on macrophages as a mechanism for hyperprogressive disease in non-small cell lung cancer subsequent to PD-1/PD-L1 blockade. Clin. Cancer Res. 25, 989-999 (2019).

13. Kato, S. et al. Hyperprogressors after immunotherapy: analysis of genomic alterations associated with accelerated growth rate. Clin. Cancer Res. 23, 4242-4250 (2017).

14. Knochelmann, H. M. et al. CAR T cells in solid tumors: blueprints for building effective therapies. Front. Immunol. 9, 1740 (2018).

15. Moon, E. K. et al. Multifactorial T-cell hypofunction that is reversible can limit the efficacy of chimeric antigen receptor-transduced human T cells in solid tumors. Clin. Cancer Res. 20, 4262-4273 (2014).

16. Rafiq, S. et al. Targeted delivery of a PD-1-blocking scFv by CAR-T cells enhances anti-tumor efficacy in vivo. Nat. Biotechnol. 36, 847-856 (2018).

17. Juneja, V. R. et al. PD-L1 on tumor cells is sufficient for immune evasion in immunogenic tumors and inhibits CD8 T cell cytotoxicity. J. Exp. Med. 214, 895-904 (2017).

18. Lin, $\mathrm{H}$. et al. Host expression of PD-L1 determines efficacy of PD-L1 pathway blockade-mediated tumor regression. J. Clin. Invest. 128, 1708 (2018).

19. Tang, H. et al. PD-L1 on host cells is essential for PD-L1 blockade-mediated tumor regression. J. Clin. Invest. 128, 580-588 (2018).

20. Lin, S.-Y. et al. Tumor PD-L1 expression and clinical outcomes in advancedstage non-small cell lung cancer patients treated with nivolumab or pembrolizumab: real-world data in Taiwan. J. Cancer 9, 1813 (2018).

21. Teixidó, C., Vilariño, N., Reyes, R. \& Reguart, N. PD-L1 expression testing in non-small cell lung cancer. Ther. Adv. Med. Oncol. 10, 1758835918763493 (2018).

22. Suarez, E. R. et al. Chimeric antigen receptor T cells secreting anti-PD-L1 antibodies more effectively regress renal cell carcinoma in a humanized mouse model. Oncotarget 7, 34341-34355 (2016).

23. Liu, M., Zhou, C. \& Zheng, J. Cigarette smoking impairs the response of EGFR-TKIs therapy in lung adenocarcinoma patients by promoting EGFR signaling and epithelial-mesenchymal transition. Am. J. Transl. Res. 7, 2026 (2015).

24. Milone, M. C. et al. Chimeric receptors containing CD137 signal transduction domains mediate enhanced survival of $T$ cells and increased antileukemic efficacy in vivo. Mol. Ther. 17, 1453-1464 (2009).

25. Huang, Q. et al. miR-153 suppresses IDO1 expression and enhances CAR T cell immunotherapy. J. Hematol. Oncol. 11, 58 (2018).

26. Zheng, Z., Chinnasamy, N. \& Morgan, R. A. Protein L: a novel reagent for the detection of chimeric antigen receptor (CAR) expression by flow cytometry. J. Transl. Med. 10, 29 (2012).

27. Herter-Sprie, G. S. et al. Synergy of radiotherapy and PD-1 blockade in Krasmutant lung cancer. JCI Insight 1, e87415 (2016).

28. Kordbacheh, T., Honeychurch, J., Blackhall, F., Faivre-Finn, C. \& Illidge, T. Radiotherapy and anti-PD-1/PD-L1 combinations in lung cancer: building better translational research platforms. Ann. Oncol. 29, 301-310 (2017).

29. Hwang, W. L. et al. Clinical outcomes in patients with metastatic lung cancer treated with PD-1/PD-L1 inhibitors and thoracic radiotherapy. JAMA Oncol. 4, 253-255 (2018).

30. Midha, A., Dearden, S. \& McCormack, R. EGFR mutation incidence in nonsmall-cell lung cancer of adenocarcinoma histology: a systematic review and global map by ethnicity (mutMapll). Am. J. Cancer Res. 5, 2892 (2015).

31. Lee, C. K. et al. Clinical and molecular characteristics associated with survival among patients treated with checkpoint inhibitors for advanced non-small cell lung carcinoma: a systematic review and meta-analysis. JAMA Oncol. 4, 210-216 (2018).

32. Yu, S., Liu, D., Shen, B., Shi, M. \& Feng, J. Immunotherapy strategy of EGFR mutant lung cancer. Am. J. Cancer Res. 8, 2106 (2018).

33. Wang, $X$. et al. Suppression of type I IFN signaling in tumors mediates resistance to anti-PD-1 treatment that can be overcome by radiotherapy. Cancer Res. 77, 839-850 (2017).

34. Dong, $\mathrm{H}$. et al. Tumor-associated B7-H1 promotes T-cell apoptosis: a potential mechanism of immune evasion. Nat. Med. 8, 793-800 (2002). 
35. Petroff, M. G., Chen, L., Phillips, T. A. \& Hunt, J. S. B7 family molecules: novel immunomodulators at the maternal-fetal interface. Placenta 23, 595-101 (2002).

36. Chen, L. \& Flies, D. B. Molecular mechanisms of T cell co-stimulation and coinhibition. Nat. Rev. Immunol. 13, 227-242 (2013).
37. Sanmamed, M. F. \& Chen, L. A paradigm shift in cancer immunotherapy: from enhancement to normalization. Cell 175, 313-326 (2018).

38. Xie, Y. J. et al. Nanobody-based CAR T cells that target the tumor microenvironment inhibit the growth of solid tumors in immunocompetent mice. Proc. Natl Acad. Sci. 116, 7624-7631 (2019). 